\title{
Dendrimersomes: a new vesicular nano-platform for MR-molecular imaging applications $\dagger$
}

Cite this: Chem. Commun., 2014 50,3453

Received 18th December 2013, Accepted 10th February 2014

DOI: $10.1039 / c 3 c c 49584 a$

www.rsc.org/chemcomm

\author{
Miriam Filippi, ${ }^{a}$ Jonathan Martinelli, ${ }^{b}$ Gilberto Mulas, ${ }^{\text {ac }}$ Marisa Ferraretto, ${ }^{a}$ \\ Eline Teirlinck, ${ }^{a}$ Mauro Botta, ${ }^{\mathrm{b}}$ Lorenzo Tei*b ${ }^{\star \mathrm{b}}$ and Enzo Terreno*ad
}

A new class of nanovesicles formed by the self-assembly of amphiphilic Janus dendrimers, dendrimersomes, loaded with hydrophilic or amphiphilic magnetic resonance imaging chelates shows promising properties as a novel, efficient and versatile nanoplatform for biomedical imaging.

Dendrimers are synthetic molecules with highly branched architectures characterized by a tree-like appearance. ${ }^{1}$ Although most dendrimers exhibit a roughly spherical symmetry, a new structural class of dendrimers has been recently obtained by joining together two chemically distinct dendritic building blocks. ${ }^{2}$ The resulting molecule is a double-faced compound with a bifunctional character, named Janus dendrimer, constituted by diverse peripheral groups on opposite sides. In particular, amphiphilic Janus dendrimers possessing one hydrophobic and one hydrophilic moiety are reminiscent of the typical structure of phospholipids, thus favouring their self-assembly in water into supramolecular aggregates of different morphologies, including nanosized bilayered vesicles named dendrimersomes. ${ }^{3-5}$ Until now, different vesicular systems such as polymersomes and liposomes have been extensively investigated for biomedical applications, mainly as nanocarriers for drug delivery or as diagnostic or theranostic agents. ${ }^{6-9}$ The main advantage associated with the use of polymersomes is their high

\footnotetext{
${ }^{a}$ Department of Molecular Biotechnology and Health Sciences,

Molecular Imaging Center, University of Torino, Via Nizza 52, 10126 Torino, Italy. E-mail: enzo.terreno@unito.it

${ }^{b}$ Dipartimento di Scienze e Innovazione Tecnologica, Università del Piemonte Orientale "Amedeo Avogadro", Viale T. Michel 11, 15121, Alessandria, Italy. E-mail: lorenzo.tei@unipmn.it

${ }^{c}$ Porto Conte Ricerche S.r.l., SP 55 Porto Conte/Capo Caccia Km 8.400, Loc Tramariglio 07041 Alghero (SS), Italy

${ }^{d}$ Center for Preclinical Imaging, University of Torino, Via Ribes 5, 10010 Colleretto Giacosa (TO), Italy. Tel: +39 0125538942/522

$\dagger$ Electronic supplementary information (ESI) available: Synthesis of a GdDOTAMAC $_{6}$-Janus-dendrimer (JD1-GdDOTAMAC ${ }_{6}$ ), the technical protocol followed for the dendrimersome assembly, DLS, stability tests in buffer, experimental information about relaxivity measurements and ${ }^{1} \mathrm{H}$ NMRD acquisition, results about quenching of the relaxivity and internalization of fluorescent probes. See DOI: $10.1039 / \mathrm{c} 3 \mathrm{cc} 49584 \mathrm{a}$
}

stability and mechanical resistance, ${ }^{10}$ while liposomes show excellent biocompatibility and marked ability to interact with natural membranes, thus providing an interesting opportunity to investigate many biological mechanisms at a cellular level. ${ }^{11}$ Although dendrimersomes should combine all these properties, ${ }^{3-5}$ their potential in biological scenarios is still completely unexplored. In particular, their use may hold promise for molecular imaging applications. ${ }^{12}$ Inspired by the versatility of liposomes that can encapsulate hydrophilic probes into the aqueous core or embed amphiphilic chelates into the phospholipid bilayer, this work aims at assessing the ability of dendrimersomes to load hydrophilic and/or amphiphilic Gd-complexes as MRI probes. The clinically approved agent Gadoteridol (GdHPDO3A) was selected as a hydrophilic probe, whilst two approaches were pursued for the incorporation of amphiphilic agents into the membrane vesicles: (i) the synthesis of a novel Janus dendrimer covalently conjugated to a Gd-chelate (JD1-GdDOTAMAC 6 ), and (ii) the incorporation into the bilayer of the lipophilic Gd-DOTAMA $\left(\mathrm{C}_{18}\right)_{2}$ complex (Scheme 1).

A large number of Janus dendrimers based on different libraries of amphiphilic molecules have been reported to form self-assembled architectures of variable sizes and morphologies. ${ }^{3}$

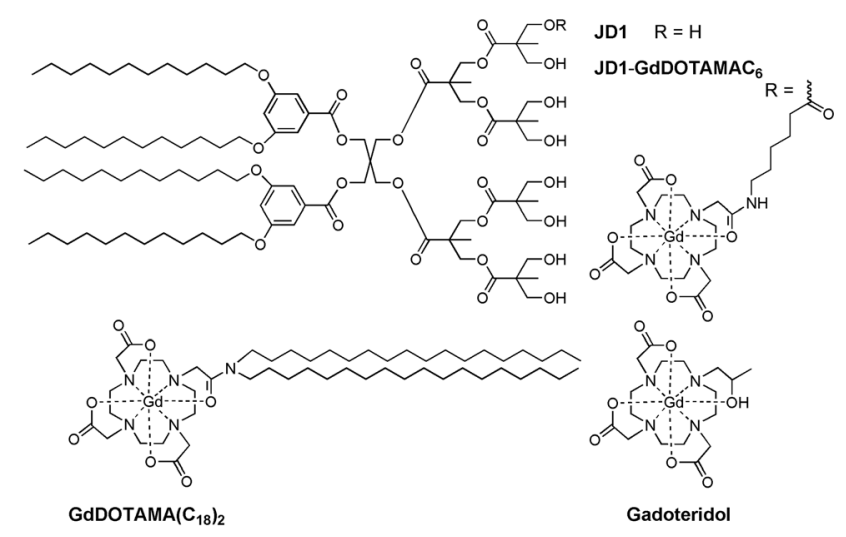

Scheme 1 Janus dendrimer JD1 and its GdDOTAMAC 6 conjugate structures (top), Gd-DOTAMA $\left(\mathrm{C}_{18}\right)_{2}$ and Gadoteridol (bottom). 
Among them, JD1 (Scheme 1) was chosen as it forms dendrimersomes with a size similar to that of liposomes ( $c a .120 \mathrm{~nm})$ and an excellent polydispersity index (PDI $=0.06) .{ }^{3}$ This is based on a pentaerythritol core with a hydrophobic moiety consisting of two 3,5-bis-dodecyl substituted benzoyl ethers and a hydrophilic segment made of one polyester dendritic structure terminating with eight hydroxyl groups. While JD1 was synthesized as described elsewhere, ${ }^{13}$ a novel synthetic procedure was followed to covalently conjugate a GdDOTA-monoamide chelate $\left(\mathrm{H}_{4}\right.$ DOTA $=1,4,7,10$-tetraazacyclododecane-1,4,7,10-tetraacetic acid) to the dendrimer. In particular, one hydroxyl group of JD1 was esterified by reaction with one equivalent of a $t$-butyl protected bifunctional chelating agent bearing an activated carboxyl function (DOTAMA $(t \mathrm{Bu})_{3^{-}}$ $\mathrm{C}_{6}$-NHS). ${ }^{14}$ The deprotection of the $t$-butyl esters, followed by complexation with $\mathrm{GdCl}_{3}$, yielded the functionalized Janus dendrimer (JD1-GdDOTAMAC 6 ; see ESI $\dagger$ for details).

Unlike most phospholipids, which easily self-organize into liposomes in physiological saline buffer, JD1 forms large, micronsized aggregates under the same isotonic conditions (see ESI $\dagger$ ). To overcome this problem, two main strategies can be followed: (i) dendrimersomes are prepared in a $5 \% \mathrm{w} / \mathrm{w}$ glucose solution, which then form vesicles with a low PDI (0.095) and a mean hydrodynamic diameter of around 100-120 nm; (ii) charged lipids (DMPG or DSPE-PEG-COOH) are incorporated into the membrane architecture to prevent the aggregation by electrostatic and/or steric repulsion. ${ }^{15-17}$ The addition of DMPG effectively allowed us to obtain a homogeneous suspension of 100-130 nm sized vesicles, but only by using phospholipids in a relatively large amount (20 mol\%). On the other hand, the addition of a small percentage (5\%) of DSPE-PEG-COOH was effective in sterically improving the stability of the nanovesicles and preventing aggregation in the isotonic buffer. Moreover, it has to be noted that PEG-2000 is normally used for coating the surface of vesicles in order to reduce their detection by the reticuloendothelial system, thus prolonging the circulatory lifetime and improving biodistribution in perfused tissues. ${ }^{15-17}$ Furthermore, the carboxylic terminal functionality of the PEG chain can serve as an anchoring site for attachment of molecules capable of modulating the biodistribution of the nanoparticle (e.g. targeting vectors).

The dendrimersomes herein reported (Fig. 1) were obtained by using the conventional film hydration method: a chloroform solution of the amphiphilic molecules was dried under vacuum

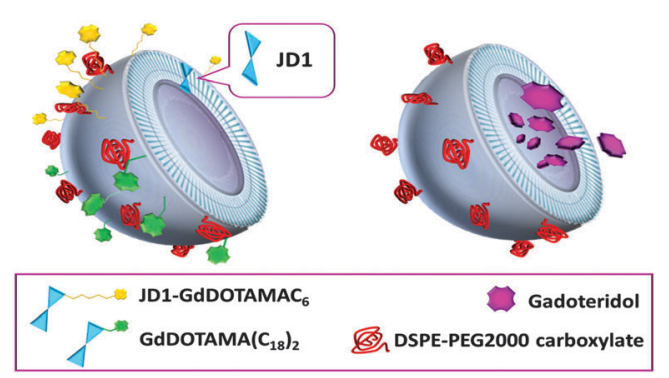

Fig. 1 Dendrimersomes made of JD1 dendrimers incorporating JD1GdDOTAMAC 6 or Gd-DOTAMA $\left(C_{18}\right)_{2}$ into the membrane bilayer (left), and encapsulating Gadoteridol in the inner core (right).
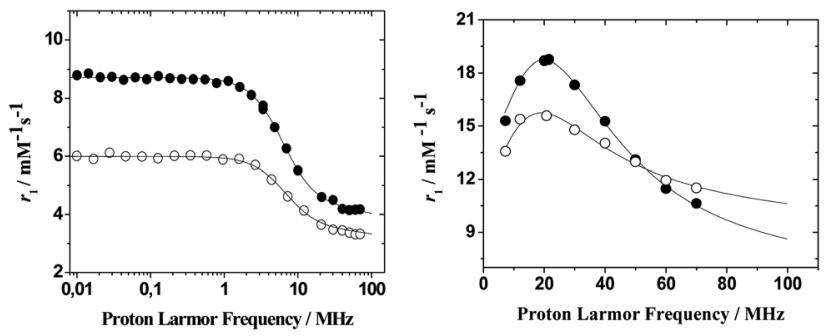

Fig. $2{ }^{1} \mathrm{H}$ NMRD profiles measured at $298 \mathrm{~K}$ of Gadoteridol (0) and dendrimersomes encapsulating Gadoteridol ( $O$ ) (left), and dendrimersomes incorporating GdDOTAMA $\left(\mathrm{C}_{18}\right)_{2}(\bullet)$ and JD1-GdDOTAMAC 6 (O) (right).

to obtain homogeneous films that were hydrated at $50{ }^{\circ} \mathrm{C}$ with an isotonic solution containing Gadoteridol $(250 \mathrm{mM})$ at $\mathrm{pH}$ 7.4. After extrusion and exhaustive dialysis, vesicles with a size ranging from 160 to $180 \mathrm{~nm}$ and a PDI lower than 0.2 were obtained (see ESI $\dagger$ ). Dendrimersomes containing Gd-complexes embedded in the lipidic bilayer were obtained by adding $20 \%$ of JD1-GdDOTAMAC 6 or Gd-DOTAMA $\left(\mathrm{C}_{18}\right)_{2}$ to the membrane formulation. ${ }^{18,19}$

The relaxometric characteristics of these nanosized systems were assessed by evaluating the magnetic field dependence of their relaxivities $\left(r_{1}\right.$, i.e. the longitudinal relaxation enhancement of the water protons induced by a $1 \mathrm{mM}$ solution of $\mathrm{Gd}^{\mathrm{III}}$ ) at $298 \mathrm{~K}$ over a wide range of field strengths (from 0.00024 to $1.65 \mathrm{~T}$, corresponding to $0.01-70 \mathrm{MHz}$ proton Larmor frequencies) to obtain the so-called nuclear magnetic relaxation dispersion (NMRD) profiles. The NMRD profiles of Gadoteridol-loaded dendrimersomes at $298 \mathrm{~K}$ (Fig. 2) exhibit the same shape of the free chelate, but with lower $r_{1}$ values over the entire range of frequency investigated. This represents a clear indication of the occurrence of a "limiting" effect on the relaxivity. It is well known that a reduced water permeability $\left(P_{\mathrm{W}}\right)$ through the vesicle bilayer results in a decrease in $r_{1}$ for the complex. ${ }^{20}$ As the observed $r_{1}$ value is only slightly lower than that observed for free Gadoteridol, we may conclude that the bilayer of dendrimersomes represents just a very weak "quenching" effect. NMRD data were analysed using the conventional Solomon-Bloembergen-Morgan (SBM) theory, ${ }^{21}$ suitably modified to take into account the encapsulation of the chelate in the nanovesicles (see ESI $\dagger$ ). The values of parameters obtained from the data analysis for the free complex were fixed during the fit of the profile of the nanovesicles using only $P_{\mathrm{W}}$ as the adjustable parameter. The value of $115 \mu \mathrm{m} \mathrm{s}^{-1}$ obtained for the water permeability (Table 1 ) is sensibly higher than those reported for conventional liposomes $\left(0.3-70 \mu \mathrm{m} \mathrm{s}^{-1}\right) .{ }^{22}$ Interestingly, the gain in $r_{1}$ due to the increase with temperature of membrane permeability seems to be compensated by the decrease of $r_{1}$ observed for free Gadoteridol (due to faster rotation), resulting in almost unchanged $r_{1}$ values for Gadoteridol-loaded dendrimersomes at $310 \mathrm{~K}$ (Fig. 3 and Fig. S5, ESI $\dagger$ ).

NMRD profiles at $298 \mathrm{~K}$ of dendrimersomes incorporating $20 \%$ of JD1-GdDOTAMAC $_{6}$ or self-assembled with $20 \%$ of Gd-DOTAMA $\left(\mathrm{C}_{18}\right)_{2}$ showed the typical shape of slowly tumbling Gd-based systems, with a peak centred at around $20 \mathrm{MHz}$ corresponding to $r_{1}$ values of 15.6 (JD1-GdDOTAMAC 6 ) and $18.7 \mathrm{mM}^{-1} \mathrm{~s}^{-1}$ (Gd-DOTAMA $\left.\left(\mathrm{C}_{18}\right)_{2}\right)$ (Fig. 2, right). Data were fitted only in the high-field region $(>3 \mathrm{MHz}$ ) because of the 
Table 1 Selected parameters obtained from the analysis of the $1 / T_{1}$ NMRD profiles at $298 \mathrm{~K}$ for dendrimersomes encapsulating Gadoteridol or incorporating GdDOTAMA $\left(\mathrm{C}_{18}\right)_{2}$ or JD1-GdDOTAMAC $6{ }^{a}$

\begin{tabular}{llll}
\hline Parameter & \multicolumn{3}{c}{ Gadoteridol GdDOTAMA $\left(\mathrm{C}_{18}\right)_{2}$ JD1-GdDOTAMAC } \\
\hline${ }^{20 \mathrm{MHz}} r_{1}\left[\mathrm{mM}^{-1} \mathrm{~s}^{-1}\right]$ & 3.65 & 18.7 & 15.6 \\
$\Delta^{2}\left[10^{19} \mathrm{~s}^{-2}\right]$ & $2.7 \pm 0.3$ & $0.64 \pm 0.05$ & $0.68 \pm 0.06$ \\
$\tau_{\mathrm{v}}[\mathrm{ps}]$ & $16.0 \pm 1.1$ & $23.0 \pm 1.3$ & $45.0 \pm 1.8$ \\
$\tau_{\mathrm{RL}}[\mathrm{ps}]$ & $65.0 \pm 7.0$ & $550 \pm 15$ & $405 \pm 12$ \\
$\tau_{\mathrm{RG}}[\mathrm{ns}]$ & - & $80 \pm 11$ & $71 \pm 9$ \\
$S^{2}$ & - & $0.65 \pm 0.1$ & $0.24 \pm 0.08$ \\
$\tau_{\mathrm{M}}[\mathrm{ns}]$ & $700 \pm 30$ & $925 \pm 40$ & $924 \pm 35$ \\
$P_{\mathrm{W}}\left[\mu \mathrm{m} \mathrm{s}^{-1}\right]$ & $115 \pm 15$ & $175 \pm 65$ & $166 \pm 60$
\end{tabular}

${ }^{a}$ The following parameters were fixed to common values during the fitting procedure: $r_{\mathrm{Gd}-\mathrm{H}}=3.0 \AA$ $, D=2.25 \times 10^{-5} \mathrm{~cm}^{2} \mathrm{~s}^{-1}, q=1, a=3.8 \AA$.

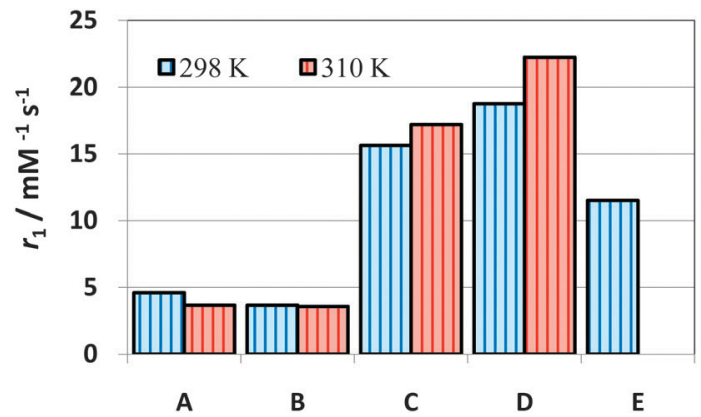

Fig. 3 Relaxivity measured at $21.5 \mathrm{MHz}, 298 \mathrm{~K}$ and $310 \mathrm{~K}$ of Gadoteridol in the free form (A) or encapsulated into dendrimersomes (B); dendrimersomes incorporated into the membrane bilayer JD1-GdDOTAMAC 6 (C) or GdDOTAMA $\left(\mathrm{C}_{18}\right)_{2}$ (D) compared to liposomes incorporating GdDOTAMA $\left(C_{18}\right)_{2}$ (E) into the lipidic bilayer.

known limitations of the SBM theory for slowly tumbling systems at low magnetic field strengths. ${ }^{21}$ We used our recently published relaxation model for the analysis of $r_{1}$ in nanovesicles incorporating paramagnetic Gd-complexes implemented using the Lipari-Szabo (LS) approach. ${ }^{19}$ The LS model was proven to be effective to treat systems in which a local molecular rotation of a chelate (characterized by a correlation time $\tau_{\mathrm{RL}}$ ) is motionally coupled to the global tumbling motion of the nanoparticle $\left(\tau_{\mathrm{RG}}\right){ }^{23}$ The motional coupling is defined by the value of the order parameter $S^{2}$ (0 = no coupling, $1=$ maximum coupling). Data were fitted by adjusting the parameters $\Delta^{2}, \tau_{\mathrm{V}}, \tau_{\mathrm{RG}}, \tau_{\mathrm{RL}}$, $\tau_{\mathrm{M}}$, and $S^{2}$, whereas $P_{\mathrm{W}}$ was left to vary only in the range of $100-250 \mu \mathrm{m} \mathrm{s}^{-1}$. On the basis of the results presented in Table 1, the lower relaxivity observed for the vesicles based on the JD1-GdDOTAMAC 6 complex with respect to those assembled with GdDOTAMA $\left(\mathrm{C}_{18}\right)_{2}$ is due to the faster rotational tumbling $\left(\tau_{\mathrm{RL}}\right)$ correlated with a poor motional coupling with the global rotation of the nanovesicle. Likely, such a difference stems from the flexible $\mathrm{C} 6$ linker that connects the coordination cage to the Janus dendrimer in JD1-GdDOTAMAC 6 . The other relaxation parameters assume the values typical of slowly tumbling GdDOTAmonoamide complexes. ${ }^{19,24}$ The $P_{\mathrm{W}}$ values obtained are slightly larger than the permeability determined for Gadoteridol-loaded dendrimersomes. However, the low statistical weight of $P_{\mathrm{W}}$ in the analysis of the profiles does not allow us to draw reliable conclusions about the role of the membrane composition in the water permeability.

At $310 \mathrm{~K}$, the relaxivity increases for both dendrimersomes incorporating Gd-amphiphilic agents, likely as a consequence of both $\tau_{\mathrm{M}}$ shortening and increased water permeability (Fig. S6, ESI $\dagger)$. Interestingly, the $\operatorname{GdDOTAMA}\left(\mathrm{C}_{18}\right)_{2}$ complex exhibits a much higher relaxivity ( $c a .50 \%$ ) when incorporated into dendrimersomes as compared to the case where it is embedded in conventional liposomal bilayers (based on DPPC, cholesterol and DSPE-PEG2000). ${ }^{19}$ This enhancement can be mostly attributed to the higher water permeability of the bilayer made of Janus dendrimers that limits the quenching effect on the relaxivity of the paramagnetic units exposed towards the inner core of the vesicles.

Finally, dendrimersomes encapsulating carboxyfluorescein at a high concentration $(20 \mathrm{mM})$ were also prepared (see $\mathrm{ESI} \dagger$ for details). They elicit a self-quenching effect of the fluorescence due to the contact and the consequent non-radiative transfer of energy among adjacent dye molecules. ${ }^{25,26}$ The quenching effect eventually vanished when breakage of vesicles by a surfactant (Triton-X100) led to probe release confirming the compartmentalization of the fluorescent dye in the aqueous core.

By virtue of their similarity to liposomes, whose versatile properties are being successfully exploited in various fields, dendrimersomes also have considerable potential for effective practical use in biomedicine. Our preliminary data indicate that the vesicles assembled from Janus dendrimers can be considered as new potential vectors of MRI agents, drugs and multimodal imaging probes. However, further studies are needed to develop and fully realize the great potential of this innovative nanosized platform.

The financial support of the "Compagnia di San Paolo" (CSP-2012 NANOPROGLY and "Validazione di molecole di tipo VHH e Aptameri per il rilascio tumore-specifico di farmaci e la valutazione contestuale della risposta mediante imaging funzionale mirato" projects), University of Torino (Innovative Nanosized Theranostic Agents), Regione Piemonte (Nano-IGT and PIIMDMT Projects), and European Union's FP7/2007-2013 under grant agreement no. HEALTH-F2-2011-278850 (INMiND) is gratefully acknowledged. This work was carried out within the framework of the COST TD1004 Action.

\section{Notes and references}

1 F. Vogtle, G. Richardt and N. Werner, Dendrimer Chemistry, Concepts, Syntheses, Properties, Applications, Wiley-VCH, Weinheim, 2009.

2 A.-M. Caminade, R. Laurent, B. Delavaux-Nicota and J.-P. Majoral, New J. Chem., 2012, 36, 217.

3 V. Percec, D. A. Wilson, P. Leowanawat, C. J. Wilson, A. D. Hughes, M. S. Kaucher, D. A. Hammer, D. H. Levine, A. J. Kim, F. S. Bates, K. P. Davis, T. P. Lodge, M. L. Klein, R. H. DeVane, E. Aqad, B. M. Rosen, A. O. Argintaru, M. J. Sienkowska, K. Rissanen, S. Nummelin and J. Ropponen, Science, 2010, 328, 1009.

4 M. Peterca, V. Percec, P. Leowanawat and A. Bertin, J. Am. Chem. Soc., 2011, 133, 20507.

5 M. Giustini, C. Bellinazzo, L. Galantini, A. Mallardi, G. Palazzo, S. Sennato, F. Bordi and K. Rissanen, Colloids Surf., A, 2012, 413, 38.

6 A. Fernandez-Fernandez, R. Manchanda and A. J. McGoron, Appl. Biochem. Biotechnol., 2011, 165, 1628.

7 R. Singh and J. W. Lillard Jr., Exp. Mol. Pathol., 2009, 86, 215.

8 T. Lammers, W. E. Hennink and G. Storm, Br. J. Cancer, 2008, 99, 392. 
9 E. Terreno, D. Delli Castelli, C. Cabella, W. Dastrù, A. Sanino, J. Stancanello, L. Tei and S. Aime, Chem. Biodiversity, 2008, 5, 1901.

10 P. Tanner, P. Baumann, R. Enea, O. Onaca, C. Palivan and W. Meier, Acc. Chem. Res., 2011, 44, 1039.

11 J. Swaminathan and C. Ehrhardt, Expert Opin. Drug Delivery, 2012, 9, 1489. 12 M. L. James and S. S. Gambhir, Physiol. Rev., 2012, 92, 897-965.

13 J. Ropponen, S. Nummelin and K. Rissanen, Org. Lett., 2004, 6, 2495.

14 S. Geninatti Crich, C. Cabella, A. Barge, S. Belfiore, C. Ghirelli, L. Lattuada, S. Lanzardo, A. Mortillaro, L. Tei, M. Visigalli, G. Forni and S. Aime, J. Med. Chem., 2006, 49, 4926.

15 D. Bhadra, S. Bhadra, P. Jain and N. K. Jain, Pharmazie, 2002, 57, 5. 16 M. C. Woodle and D. D. Lasic, Biochim. Biophys. Acta, 1992, 1113, 171. 17 M. L. Immordino, F. Dosio and L. Cattel, Int. J. Nanomed., 2006, 1, 297.

18 G. A. Rolla, M. Botta, L. Tei, C. Cabella, S. Ghiani, C. Brioschi and A. Maiocchi, Chem.-Eur. J., 2013, 19, 11189.
19 E. Cittadino, M. Botta, L. Tei, F. Kielar, R. Stefania, E. Chiavazza, S. Aime and E. Terreno, ChemPlusChem, 2013, 78, 712.

20 S. Laurent, L. V. Elst, C. Thirifays and R. N. Muller, Langmuir, 2008, 24, 8.

21 The Chemistry of Contrast Agents in Medical Magnetic Resonance Imaging, ed. A. Merbach, L. Helm and E. Toth, John Wiley \& Sons Ltd, 2nd edn, 2013.

22 E. Terreno, A. Sanino, C. Carrera, D. Delli Castelli, G. B. Giovenzana, A. Lombardi, R. Mazzon, L. Milone, M. Visigalli and S. Aime, J. Inorg. Biochem., 2008, 102, 5-6.

23 (a) G. Lipari and S. Szabo, J. Am. Chem. Soc., 1982, 104, 4546;

(b) G. Lipari and S. Szabo, J. Am. Chem. Soc., 1982, 104, 4559.

24 M. Botta and L. Tei, Eur. J. Inorg. Chem., 2012, 1945.

25 R. F. Chen and J. R. Knutson, Anal. Biochem., 1988, 172, 61.

26 M. Sila, S. Au and N. Weiner, Biochim. Biophys. Acta, 1986, 859. 